\title{
One-Pot Synthesis of Substituted Coumarins Catalyzed by Silica Gel Supported Sulfuric Acid Under Solvent-Free Conditions
}

\author{
Benjaram M. Reddy ${ }^{*}$, Boningari Thirupathi and Meghshyam K. Patil \\ Inorganic and Physical Chemistry Division, Indian Institute of Chemical Technology, Uppal Road, Hyderabad - 500 \\ 607, India
}

\begin{abstract}
A remarkable acceleration in the synthesis of substituted coumarins via Pechmann reaction catalyzed by silica gel supported sulfuric acid $\left(\mathrm{H}_{2} \mathrm{SO}_{4} /\right.$ silica gel) at $120{ }^{\circ} \mathrm{C}$ in high yields under solvent-free reaction condition with short reaction times is described. This methodology offers momentous improvements over various options for the synthesis of coumarins with regard to yield of products, simplicity in operation and green aspects by avoiding toxic catalysts and solvents.
\end{abstract}

Keywords: Pechmann reaction, phenols, reaction mechanism, Coumarin derivatives, $\mathrm{H}_{2} \mathrm{SO}_{4} /$ silica gel, solvent-free.

\section{INTRODUCTION}

Coumarin and its derivatives form an important class of benzopyrones found in Nature. They are structural subunits in many complex natural products and have shown numerous biological activities, such as antitumor [1], anti-HIV (NNRTI) [2], antioxidation [3], tumor necrosis factor-a (TNF-a) inhibition [4], antimicrobial activity [5], serine protease inhibition [6] and anticancer activity [7]. The widespread biological activities of coumarin derivatives have aroused great interest in the area of synthetic chemistry and pharmacology.

Coumarins could be synthesized by various methods, such as Pechmann [8], Perkin [9], Knoevenagel [10], Reformatsky [11], Witting [12], Claisen [13] and flash vacuum pyrolysis reaction [14]. However, the Pechmann reaction is one of the simplest and direct methods for the synthesis of coumarins since it proceeds from very simple starting materials, namely, phenols and $\beta$-keto esters or $\alpha, \beta$-unsaturated carboxylic acids utilizing various catalysts, such as sulfuric acid [8], trifluoroacetic acid [15], phosphorus pentoxide [16], $\mathrm{ZrCl}_{4}$ [17], $\mathrm{TiCl}_{4}$ [18] and ionic liquids [19]. However, most of these procedures require long duration $(24 \mathrm{~h} \mathrm{[20],20 \textrm {h }}$ [21]), high temperature $\left(150^{\circ} \mathrm{C}\right)[21]$ and also microwave irradiation [22]. Also there have been some attempts to find alternative, environmentally benign synthesis routes. On those lines, Nafion-H [23], zeolite H-BEA, modified zirconia [24], Amberlyst 15 [25], montmorillonite clay [26] and other solid acids have been employed for this purpose in the Pechmann condensation.

It has been realized in recent years that sulfuric acid adsorbed on silica gel could be used as a multipurpose acid catalyst [27-31]. In continuation of our work on the development of useful synthetic methodologies by employing solid acid catalysts [32-35], we observed that $\mathrm{H}_{2} \mathrm{SO}_{4} /$ silica

\footnotetext{
*Address correspondence to this author at the Inorganic and Physical Chemistry Division, Indian Institute of Chemical Technology, Uppal Road, Hyderabad - 500 607, India;

E-mails: bmreddy@iict.res.in, mreddyb@yahoo.com
}

gel is an efficient catalyst for the synthesis of coumarins via Pechmann condensation. In this communication, we are disclosing our findings on $\mathrm{H}_{2} \mathrm{SO}_{4} /$ silica gel catalyzed Pechmann condensation of a series of phenols and $\beta$-keto esters to coumarins under solvent free conditions.

\section{EXPERIMENTAL}

\section{Preparation of $\mathrm{H}_{2} \mathrm{SO}_{4} /$ Silica Gel}

A solution of $1 \mathrm{~mL}$ conc. $\mathrm{H}_{2} \mathrm{SO}_{4}$ in $15 \mathrm{~mL}$ acetone is added to a dispersion of $50 \mathrm{~g}$ silica gel (Merck 230-400 mesh, $60 \AA$ ) in $100 \mathrm{~mL}$ acetone and stirred at room temperature for $1 \mathrm{~h}$. The solvent was removed under reduced pressure. A yellow-brown powder was obtained, which can be stored in a desiccator for long periods of time without any appreciable loss of activity.

\section{Typical Reaction Procedure}

In a typical experiment, $\mathrm{H}_{2} \mathrm{SO}_{4} /$ silica gel $(100 \mathrm{mg})$ was dispersed in a mixture of phenols $(2 \mathrm{mmol})$ and $\beta$ - keto esters $(2.4 \mathrm{mmol})$ in a $25-\mathrm{mL}$ batch reactor equipped with a distillation condenser. The content was stirred vigorously at $120^{\circ} \mathrm{C}$. The progress of the reaction was monitored by TLC. At the end of the reaction, the reaction mixture was treated with $\mathrm{CH}_{3} \mathrm{OH}$ and filtered to recover the catalyst. It was reused for several times without loss of activity. The filtrate was evaporated under reduced pressure to obtain the crude product. Thus obtained product was washed with water, filtered and dried at $100{ }^{\circ} \mathrm{C}$. The product was purified by dissolving in $20 \mathrm{~mL} 1 \mathrm{M} \mathrm{NaOH}$ and then regenerated with $10 \mathrm{~mL} 2 \mathrm{M} \mathrm{H}_{2} \mathrm{SO}_{4}$ solution. NMR, IR and mass spectroscopic techniques were used to analyze the products and compared with the authentic samples.

\section{RESULTS AND DISCUSSION}

The reaction of phenols and $\beta$-keto esters in the presence of catalytic amount of $\mathrm{H}_{2} \mathrm{SO}_{4} /$ silica gel afforded the corresponding coumarin derivatives (Scheme 1). The reactions were clean and affording exclusively coumarins in high yields in a relatively short times. It is an established fact in the literature that Pechmann reaction proceeds through trans- 
<smiles>[R]C(=O)C([Z7])C(=O)OCC(C)C(C)C(C)C</smiles>

Scheme 1. Pechmann condensation reaction of phenols with $\beta$-keto esters to substituted coumarins.<smiles>[R3]C1=CC(O)=CC(=O)CC1[R]</smiles>

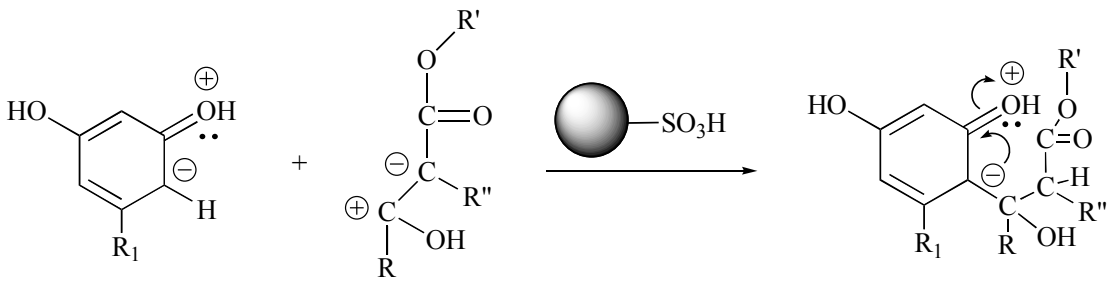

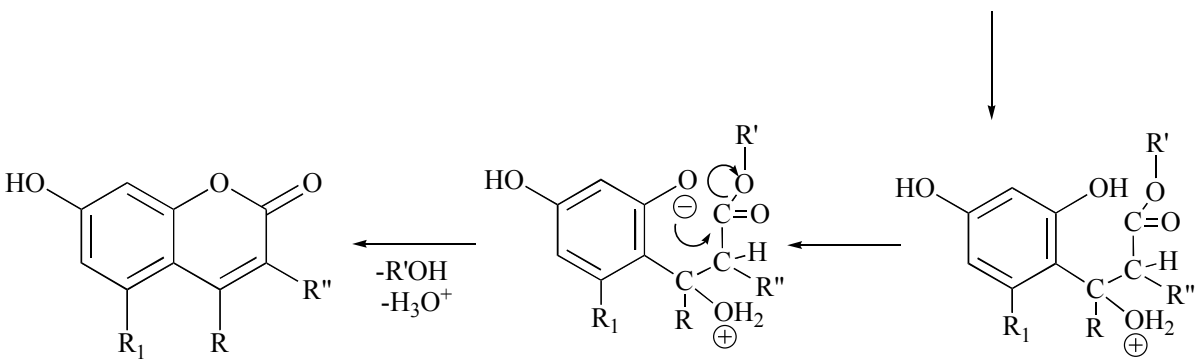

Scheme 2. Plausible mechanism for the pechmann condensation of phenols and $\beta$-keto esters by $\mathrm{H}_{2} \mathrm{SO}_{4} /$ silica gel.

esterification and intramolecular hydroxyalkylation, followed by dehydration $[18,24]$. These three steps are all typical acid-catalyzed reactions. Therefore, the outcome of the Pechmann reaction depends very much on the Brønsted acidity of the catalysts [16]. We have given a plausible mechanism for the Pechmann condensation of phenols and $\beta$-keto esters by $\mathrm{H}_{2} \mathrm{SO}_{4} /$ silica gel in Scheme 2 .

To optimize the reaction conditions such as temperature, solvent and amount of catalyst $\left(\mathrm{H}_{2} \mathrm{SO}_{4} /\right.$ silica gel), we selected the highest time taking reaction that is the reaction between orcinol and ethyltrifluoroacetoacetate as a model reaction. The results are collected in Table $\mathbf{1}$. Lower catalytic activity is observed for various organic solvents such as toluene, $\mathrm{MeOH}, \mathrm{CH}_{2} \mathrm{Cl}_{2}$ under reflux condition and $50 \mathrm{mg}$ $\mathrm{H}_{2} \mathrm{SO}_{4} /$ silica gel catalyst, which probably due to interference of solvent with active site of the catalyst (Table 1, entry 6-8). On the contrary, reaction worked well under solvent-free condition (Table 1, Entry 1-5). On increasing reaction temperature from 60 to $120^{\circ} \mathrm{C}$, product yield and time were found to be more favorable at $120^{\circ} \mathrm{C}$ (Table 1, entry 1-4). Again increasing the catalyst amount the reaction moved faster (Table 1, entry $\mathbf{1 , 5}$ ). Interestingly, no reaction took place in the absence of catalyst for 2 days of reaction time.

The sulfated silica gel solid acid catalyst was evaluated for liquid phase synthesis of coumarins in solvent free conditions and summarized in Table 2. Different types of $\beta$-keto esters were used namely, ethyl acetoacetate (Table 2, entries 1-6 and 20), methyl acetoacetate (Table 2, entries 7-10), methyl cyclohexanone-2-corboxylate (Table 2, entries 1113), ethyl trifluoro acetoacetate (Table 2 , entries 14, 15) and $\alpha$-methyl ethyl acetoacetate (Table 2, entries 16-19). Reactions of phloroglucinol with ethyl acetoacetate, methyl acetoacetate and $\alpha$-methyl ethyl acetoacetate (Table $\mathbf{2}$, entries $\mathbf{4}$, 10, 19) took place very fast within 3,3 and $18 \mathrm{~min}$, respectively. This is mainly due to the presence of three hydroxyl groups that cooperate in activating the aromatic ring for hydroxyalkylation. Similarly, reactions of pyrogallol with ethyl acetoacetate, methyl acetoacetate and $\alpha$-methyl ethyl acetoacetate (Table 2, entries 3,9, 18) took place in 6,7 and 26 $\mathrm{min}$, respectively, which are slower than that of the reactions of phloroglucinol presumably due to steric hindrance of hydroxyl groups. Again reaction of pyrogallol with methyl cyclohexanone-2-corboxylate was completed within $3 \mathrm{~min}$. The reactions of resorcinol with ethyl acetoacetate, methyl acetoacetate, $\alpha$-methyl ethyl acetoacetate, methyl cyclohexanone-2-corboxylate and ethyl trifluoro acetoacetate (Table 2, entries 2, 8, 17, 12 and 15) occurred within 5, 7, 10, 5 and $65 \mathrm{~min}$, respectively. This could be due to the presence of only two hydroxyl groups, which are meta to each other. However, in the case of orcinol, the reactions (Table 2, entries 1, 7, 16, 11 and 14) were somewhat slower than that of the reactions of resorcinol that could be due to the steric hindrance of methyl group ortho to the position of hydroxyalkylation. Table 1 compares the activities of various $\beta$-keto esters with various phenols, Reactivity order for $\beta$-keto esters is found to be ethyl acetoacetate $\approx$ methyl acetoace- 
Table 1. Effect of Temperature, Solvent, Amount of Catalyst $\left(\mathrm{H}_{2} \mathrm{SO}_{4} /\right.$ Silica Gel) on the Synthesis of Coumarins<smiles>CCOC(=O)CC(=O)C(F)(F)F</smiles>

\begin{tabular}{|c|c|c|c|c|c|}
\hline S. No. & Catalyst Amount (mg) & Oil Bath Temperature $\left({ }^{\circ} \mathrm{C}\right)$ & Solvent & Time $\left(\min ^{\mathrm{c}}\right),\left(\mathrm{hrs}^{\mathrm{d}}\right),\left(\right.$ Days $\left.^{\mathrm{e}}\right)$ & Yield $(\%)^{\mathrm{b}}$ \\
\hline 1. & 50 & 120 & - & $110^{\mathrm{c}}$ & 73 \\
\hline 2. & 50 & 100 & - & $5.5^{\mathrm{d}}$ & 71 \\
\hline 3. & 50 & 80 & - & $11^{\mathrm{d}}$ & 78 \\
\hline 4. & 50 & 60 & - & $16^{\mathrm{d}}$ & 85 \\
\hline 5. & 100 & 120 & - & $70^{\mathrm{c}}$ & 87 \\
\hline 6. & 50 & 120 & Toluene & $20^{\mathrm{d}}$ & 75 \\
\hline 7. & 50 & 120 & $\mathrm{CH}_{3} \mathrm{OH}$ & $24^{\mathrm{d}}$ & 60 \\
\hline 8. & 50 & 120 & $\mathrm{CH}_{2} \mathrm{Cl}_{2}$ & $24^{\mathrm{d}}$ & 62 \\
\hline 9. & - & 120 & - & $02^{\mathrm{e}}$ & NR \\
\hline
\end{tabular}

tate $>$ methyl cyclohexanone-2-corboxylate $>\alpha$-methyl ethyl acetoacetate $>$ ethyl trifluoro acetoacetate. The o-hydroxy phenol and m-methoxy phenol (Table 2, entry 5, 6 Table 1) are found to be inactive and gallic acid (Table $\mathbf{2}$, entry $\mathbf{2 0}$ ) is also less active may be due to steric hindrance by acid $(\mathrm{COOH})$ group to the position of ortho to hydroxyalkylation. Interestingly, with $\mathrm{H}_{2} \mathrm{SO}_{4}$ /silica gel catalyst a $95 \%$ yield was obtained in just $3 \mathrm{~min}$. All products were characterized by comparison of their ${ }^{1} \mathrm{H}$ NMR, mass and IR spectra with those of authentic samples, and then we conclude that selectively one product (A) is forming during the reaction (Scheme 3 ). The spectral data for some selected representative compounds are given below:

\section{7-Hydroxy-4, 5-dimethyl-chromen-2-one (Entry 1, Table 2)}

${ }^{1} \mathrm{HNMR}\left(\mathrm{CDCl}_{3}+\mathrm{DMSO}, 200 \mathrm{MHz}\right): \delta 9.85(\mathrm{bs}, 1 \mathrm{H}$, $\mathrm{OH}), 6.51$ (s, 2H, aromatic protons), 5.88 (s, 1H, allylic $\mathrm{CH})$, 2.97 (attributable to residual water in product), $2.58(\mathrm{~s}, 3 \mathrm{H}$, Me), 2.30 (s, 3H, Me) ppm. IR (Neat): v 3251, 1627, 1443, 1379, 1133, 1080, 908, 681, $535 \mathrm{~cm}^{-1}$. EIMS: $m / z-190$ $\left[\mathrm{M}^{+}\right]$.

\section{7-Hydroxy-4-methyl-chromen-2-one (Entry 2, Table 2)}

${ }^{1} \mathrm{HNMR}\left(\mathrm{CDCl}_{3}+\mathrm{DMSO}, 200 \mathrm{MHz}\right): \delta 7.36-7.41(\mathrm{q}, \mathrm{J}$ $=6.00, \mathrm{~J}=1.50,1 \mathrm{H}$, aromatic proton $), 6.71-6.76(\mathrm{~m}, 2 \mathrm{H}$, aromatic protons), $5.99(\mathrm{~d}, \mathrm{~J}=1.50,1 \mathrm{H}$, allylic $\mathrm{CH}), 2.37(\mathrm{~d}$, $\mathrm{J}=1.50,3 \mathrm{H}, \mathrm{Me}$ ) ppm. IR (Neat): v 3155, 1678, 1400, 1227 , 1057, 974, 844, $748 \mathrm{~cm}^{-1}$. EIMS: $m / z-176\left[\mathrm{M}^{+}\right]$.

\section{7,8-Dihydroxy-4-methyl-chromen-2-one (Entry 3, Table 2)}

${ }^{1} \mathrm{HNMR}\left(\mathrm{CDCl}_{3}+\mathrm{DMSO}, 200 \mathrm{MHz}\right): \delta 6.96(\mathrm{~d}, \mathrm{~J}=8.81$ $\mathrm{Hz}, 1 \mathrm{H}$, aromatic proton), $6.78(\mathrm{~d}, \mathrm{~J}=8.81 \mathrm{~Hz}, 1 \mathrm{H}$, aromatic proton), 5.99 (s, 1H, olefinic $\mathrm{CH}), 2.38(\mathrm{~s}, 3 \mathrm{H}, \mathrm{Me}) \mathrm{ppm}, \mathrm{OH}$ is unobserved. IR (Neat): v 3424, 1842, 1660, 1593, 1450,
1332, 1276, 1072, 910, 804, $504 \mathrm{~cm}^{-1}$. EIMS: $m / z-192$ $\left[\mathrm{M}^{+}\right]$.

5,7-Dihydroxy-4-methyl-chromen-2-one (Entry 4, Table 2)

${ }^{1} \mathrm{NMR}\left(\mathrm{CDCl}_{3}+\mathrm{DMSO}, 200 \mathrm{MHz}\right): \delta 6.24(\mathrm{~d}, \mathrm{~J}=2.19$ $\mathrm{Hz}, 1 \mathrm{H}$, aromatic proton), $6.19(\mathrm{~d}, \mathrm{~J}=2.19 \mathrm{~Hz}, 1 \mathrm{H}$, aromatic proton), 5.70 (s, 1H, olifinic proton), 2.11 (s, 3H, Me) ppm, $\mathrm{OH}$ is unobserved. IR (Neat): $v 3447,1865,1660,1618$, $1530,1456,1160,815,750,570 \mathrm{~cm}^{-1}$. EIMS: $m / z-192$ $\left[\mathrm{M}^{+}\right]$.

7-Hydroxy-4, 5-dimethyl-chromen-2-one (Entry 7, Table 2)

${ }^{1} \mathrm{HNMR}\left(\mathrm{CDCl}_{3}+\mathrm{DMSO}, 200 \mathrm{MHz}\right): \delta 10.02(\mathrm{bs}, 1 \mathrm{H}$, $\mathrm{OH}) 6.52(\mathrm{~s}, 2 \mathrm{H}$, aromatic protons $), 5.89(\mathrm{~s}, 1 \mathrm{H}$, allylic proton), 3.24 (attributable to residual water in product) $2.58(\mathrm{~s}$, 3H, Me), 2.31 (s, 3H, Me) ppm. IR (Neat): v 3251, 1627, 1443, 1379, 1133, 1080, 908, 681, $535 \mathrm{~cm}^{-1}$. EIMS: $m / z-$ $190\left[\mathrm{M}^{+}\right]$.

\section{7-Hydroxy-4-methyl-chromen-2-one (Entry 8, Table 2)}

${ }^{1} \mathrm{HNMR}\left(\mathrm{CDCl}_{3}+\mathrm{DMSO}, 200 \mathrm{MHz}\right): \delta 9.90(\mathrm{bs}, 1 \mathrm{H}$, OH) $7.37-7.41(\mathrm{q}, \mathrm{J}=1.46, \mathrm{~J}=0.73,1 \mathrm{H}), 6.70-6.76(\mathrm{~m}$, $2 \mathrm{H}), 5.98(\mathrm{~d}, \mathrm{~J}=1.46,1 \mathrm{H}), 3.08$ (attributable to residual water in product) $2.37(\mathrm{~d}, \mathrm{~J}=1.46,3 \mathrm{H}, \mathrm{Me}) \mathrm{ppm}$. IR (Neat): 3160, 1658, 1380, 1242, 1024, 996, 856, 787, $666 \mathrm{~cm}^{-1}$. EIMS: $m / z-176\left[\mathrm{M}^{+}\right]$.

\section{7,8-Dihydroxy-4-methyl-chromen-2-one (Entry 9, Table} 2)

${ }^{1} \mathrm{HNMR}\left(\mathrm{CDCl}_{3}+\mathrm{DMSO}, 200 \mathrm{MHz}\right): \delta 6.97(\mathrm{~d}, J=8.81$ $\mathrm{Hz}, 1 \mathrm{H}$, aromatic proton), $6.77(\mathrm{~d}, J=8.81 \mathrm{~Hz}, 1 \mathrm{H}$, aromatic proton), 5.99 (s, 1H, olefinic proton), 3.59 (attributable to residual water in product), $2.37(\mathrm{~s}, 3 \mathrm{H}, \mathrm{Me}) \mathrm{ppm}, \mathrm{OH}$ is unobserved. IR (Neat): $v$ 3420, 1839, 1597, 1447, 1325, 1270, 1061, 901, 784, $515 \mathrm{~cm}^{-1}$. EIMS: $\mathrm{m} / z-192\left[\mathrm{M}^{+}\right]$. 
Table 2. $\mathrm{H}_{2} \mathrm{SO}_{4} /$ Silica Gel Catalyzed Pechmann Condensation in Solvent Free Conditions

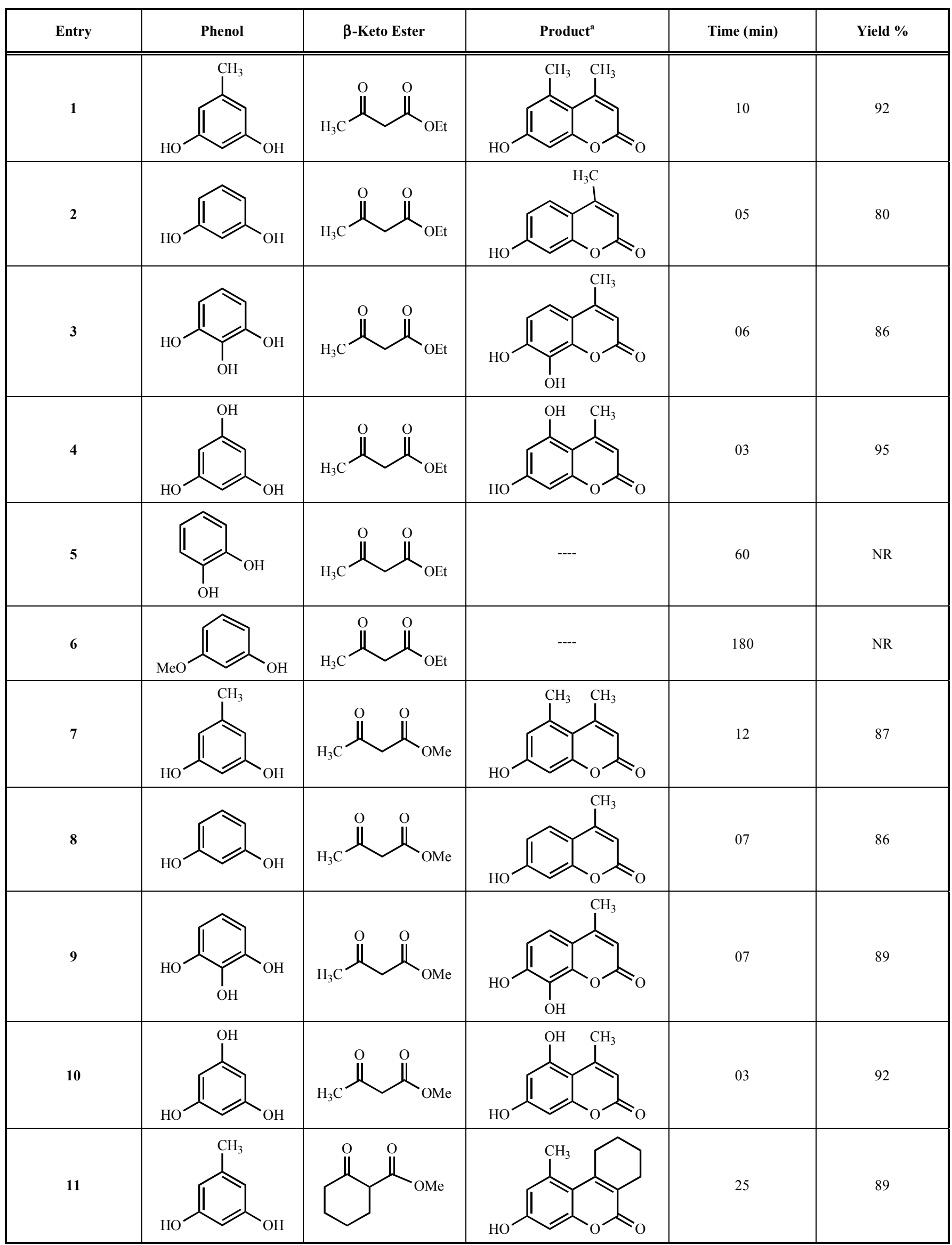




\begin{tabular}{|c|c|c|c|c|c|}
\hline Entry & Phenol & $\beta$-Keto Ester & Product $^{a}$ & Time (min) & Yield \% \\
\hline 12 & & & & 05 & 91 \\
\hline 13 & & & & 03 & 91 \\
\hline 14 & & & & 110 & 73 \\
\hline 15 & & & & 65 & 81 \\
\hline 16 & & & & 40 & 75 \\
\hline 17 & & & & 10 & 82 \\
\hline 18 & & & & 26 & 78 \\
\hline 19 & & & & 18 & 85 \\
\hline 20 & & & -- & 90 & NR \\
\hline
\end{tabular}

a The structures of the products were determined from their spectroscopic (1H NMR and MS) data

${ }^{\mathrm{b}}$ Isolated yield.

\section{5,7-Dihydroxy-4-methyl-chromen-2-one (Entry 10, Table 2)}

${ }^{1} \mathrm{NMR}\left(\mathrm{CDCl}_{3}+\mathrm{DMSO}, 200 \mathrm{MHz}\right): \delta 6.24(\mathrm{~d}, \mathrm{~J}=2.19 \mathrm{~Hz}$, $1 \mathrm{H}$, aromatic proton), $6.18(\mathrm{~d}, J=2.19 \mathrm{~Hz}, 1 \mathrm{H}$, aromatic proton), 5.71 (s, 1H, olifinic proton), $2.54(\mathrm{~s}, 3 \mathrm{H}, \mathrm{Me}) \mathrm{ppm}, \mathrm{OH}$ is unobservered. IR (Neat): v 3473, 1876, 1660, 1618, 1533, 1416, $1156,815,723,646,582 \mathrm{~cm}^{-1}$. EIMS: $m / z-192\left[\mathrm{M}^{+}\right]$.
3-Hydroxy-1-methyl-7,8,9,10-tetrahydro-benzo[c]chromen-6one (Entry 11, Table 2)

${ }^{1} \mathrm{NMR}\left(\mathrm{CDCl}_{3}+\mathrm{DMSO}, 200 \mathrm{MHz}\right): \delta 9.84(\mathrm{~s}, 1 \mathrm{H}, \mathrm{OH})$, 6.49 (s, 2H, aromatic protons), 2.98- $3.13(\mathrm{~m}, 2 \mathrm{H}$, cyclohexyl), 2.36- 2.51 (m, 2H, cyclohexyl), 2.29 (s, 3H, Me) 1.62- 1.83 (m, 4H, cyclohexyl) ppm. IR (KBr): v 3222, 
<smiles>[R]OC(=O)C([R7])C([R])=O</smiles><smiles>[R]c1c([R])c2c(C)cc(O)cc2oc1=O</smiles>

A<smiles>[R]c1c([R])c2c(O)cc(C)cc2oc1=O</smiles>

B

Scheme 3. Selectivity of the product for the pechmann condensation.

2933, 2861, 1667, 1600, 1267, 1091, 826, 737, $527 \mathrm{~cm}^{-1}$. EIMS: $m / z-230\left[\mathrm{M}^{+}\right]$.

\section{3-Hydroxy-7,8,9,10-tetrahydro-benzo[c]chromen-6-one (Entry 12, Table 2)}

${ }^{1} \mathrm{NMR}\left(\mathrm{CDCl}_{3}+\mathrm{DMSO}, 200 \mathrm{MHz}\right): \delta 9.79(\mathrm{~s}, 1 \mathrm{H}, \mathrm{OH})$, 7.30- 7.40 (m, $1 \mathrm{H}$, aromatic proton $), 6.64-6.73(\mathrm{~m}, 1 \mathrm{H}$, aromatic protons), 3.02 (due to water remaining in product), 2.67- 2.81 (m, 2H, cyclohexyl), 2.38- 2.55 (m, 2H, cyclohexyl), 1.69- $1.84(\mathrm{~m}, 4 \mathrm{H}$, cyclohexyl) ppm. IR (KBr): v 3214, 2937, 1678, 1613, 1564, 1151, 1102, 1040, 852, 754, $497 \mathrm{~cm}^{-1}$. EIMS: $\mathrm{m} / z-216\left[\mathrm{M}^{+}\right]$.

\section{3,4-Dihydroxy-7, 8, 9, 10-tetrahydro-benzo[c]chromen-6-} one (Entry 13, Table 2)

${ }^{1} \mathrm{NMR}\left(\mathrm{CDCl}_{3}+\mathrm{DMSO}, 200 \mathrm{MHz}\right): \delta 9.09$ (bs, 2H, OH), $6.90(\mathrm{~d}, J=8.32 \mathrm{~Hz}, 1 \mathrm{H}$, aromatic proton), $6.73(\mathrm{~d}, J=8.32$ $\mathrm{Hz}, 1 \mathrm{H}$, aromatic proton), 3.02 (due to water remaining in product), 2.66- $2.84(\mathrm{~m}, 2 \mathrm{H}$, cyclohexyl), 2.40- $2.60(\mathrm{~m}, 2 \mathrm{H}$, cyclohexyl), 1.63- 1.98 (m, 4H, cyclohexyl) ppm. IR (KBr): v 3464, 2946, 1674, 1616, 1580, 1508, 1382, 1318, 1095, 975, 807, $762 \mathrm{~cm}^{-1}$. EIMS: m/z - $232\left[\mathrm{M}^{+}\right]$.

\section{7-Hydroxy-5-methyl-4-trifluoromethyl-chromen-2-one (Entry 14, Table 2)}

${ }^{1} \mathrm{NMR}\left(\mathrm{CDCl}_{3}+\mathrm{DMSO}, 200 \mathrm{MHz}\right): 10.52(\mathrm{~s}, 1 \mathrm{H}, \mathrm{OH}$ proton), 6.54-6.66 ( $\mathrm{m}, 3 \mathrm{H}$, aromatic protons), 3.17 (due to water remaining in product), $2.34\left(\mathrm{~s}, 3 \mathrm{H}, \mathrm{CH}_{3}\right) \mathrm{ppm}$. IR $(\mathrm{KBr}): \mathrm{v} 3420,3162,2955,1725,1618,1512,1309,1213$, 1161, 926, 863, 695, 656, $539 \mathrm{~cm}^{-1}$. EIMS: $m / z-244\left[\mathrm{M}^{+}\right]$.

\section{7-Hydroxy-4-trifluoromethyl-chromen-2-one (Entry 15,} Table 2)

${ }^{1} \mathrm{NMR}\left(\mathrm{CDCl}_{3}+\mathrm{DMSO}, 200 \mathrm{MHz}\right)$ : one broad singlet observed at $\delta 10.58,7.46-7.52(\mathrm{dd}, J=6.25 \mathrm{~Hz}, J=2.34 \mathrm{~Hz}$, $1 \mathrm{H}$, aromatic proton), 6.75-6.90 ( $\mathrm{m}, 2 \mathrm{H}$, aromatic protons), $6.51(\mathrm{~s}, 1 \mathrm{H}$, allylic proton) 3.30 (due to water remaining in product) ppm. IR (KBr): v 3399, 3104, 2921, 1714, 1609, $1405,1286,1193,1131,891,857,655,624,498 \mathrm{~cm}^{-1}$. EIMS: $m / z-230\left[\mathrm{M}^{+}\right]$.

\section{7-Hydroxy-3,4,5-trimethyl-chromen-2-one (Entry 16,} Table 2)

${ }^{1} \mathrm{NMR}\left(\mathrm{CDCl}_{3}+\mathrm{DMSO}, 200 \mathrm{MHz}\right): \delta 9.78(\mathrm{bs}, 1 \mathrm{H}, \mathrm{OH})$, $6.51(\mathrm{~s}, 2 \mathrm{H}$, aromatic protons), 3.10 (due to water) 2.60 (s, 3H, Me), 2.29 (s, 3H, Me), 2.09 (s, 3H, Me) ppm. IR (KBr): v 3210,1673, 1618, 1281, 1117, 1061, 827, 745, $538 \mathrm{~cm}^{-1}$. EIMS: $m / z-204\left[\mathrm{M}^{+}\right]$.

\section{7-Hydroxy-3,4,-dimethyl-chromen-2-one (Entry 17, Ta-} ble 2)

${ }^{1} \mathrm{NMR}\left(\mathrm{CDCl}_{3}+\mathrm{DMSO}, 200 \mathrm{MHz}\right): \delta 7.39(\mathrm{~d}, J=8.08$ $\mathrm{Hz}, 1 \mathrm{H}$, aromatic proton), 6.64- 6.78 (m, $2 \mathrm{H}$, aromatic pro- tons), $2.34(\mathrm{~s}, 3 \mathrm{H}, \mathrm{Me}), 2.11(\mathrm{~s}, 3 \mathrm{H}, \mathrm{Me}) \mathrm{ppm}, \mathrm{OH}$ is unobserverved. IR (KBr): v 3189, 1677, 1616, 1566, 1319, 1236, 1149, 1097, 857, 762, $730 \mathrm{~cm}^{-1}$ EIMS: $m / z-190\left[\mathrm{M}^{+}\right]$.

7,8-Dihydroxy-3,4,-dimethyl-chromen-2-one (Entry 18, Table 2)

${ }^{1} \mathrm{NMR}\left(\mathrm{CDCl}_{3}+\mathrm{DMSO}, 200 \mathrm{MHz}\right): \delta 6.96(\mathrm{~d}, J=8.81$ $\mathrm{Hz}, 1 \mathrm{H}$, aromatic proton), $6.75(\mathrm{~d}, J=8.81 \mathrm{~Hz}, 1 \mathrm{H}$, aromatic proton), $2.35(\mathrm{~s}, 3 \mathrm{H}, \mathrm{Me}), 2.13(\mathrm{~s}, 3 \mathrm{H}, \mathrm{Me}) \mathrm{ppm}, \mathrm{OH}$ is unobserverved. IR $(\mathrm{KBr})$ : v 3425, 3065, 1673, 1581, 1347, 1303, 1109, 816, $764 \mathrm{~cm}^{-1}$. EIMS: $\mathrm{m} / \mathrm{z}-206\left[\mathrm{M}^{+}\right]$.

5,7-Dihydroxy-3，4,-dimethyl-chromen-2-one (Entry 19, Table 2)

${ }^{1} \mathrm{NMR}\left(\mathrm{CDCl}_{3}+\mathrm{DMSO}, 200 \mathrm{MHz}\right): \delta 9.75(\mathrm{~s}, 1 \mathrm{H}), 6.20$ (dd, $J=9.06 \mathrm{~Hz}, \mathrm{~J}=3.02 \mathrm{~Hz}, 2 \mathrm{H}$, aromatic protons), 2.56 (s, $3 \mathrm{H}, \mathrm{Me}), 2.06$ (s, 3H, Me), $1.24(\mathrm{~s}, 1 \mathrm{H}) \mathrm{ppm}$. IR (KBr): v 3269, 2920, 1675, 1615, 1561, 1383, 1358, 1285, 1167, 1056, 836, 742, $559 \mathrm{~cm}^{-1}$. EIMS: $m / z-206\left[\mathrm{M}^{+}\right]$.

\section{CONCLUSION}

In summary, a convenient method has been developed for the Pechmann reaction of phenols and $\beta$-keto esters catalyzed by $\mathrm{H}_{2} \mathrm{SO}_{4} /$ silica gel. Use of inexpensive and reusable catalyst, solvent-free condition, short reaction time, high yield and ease of purification of the product are the key features of this elegant protocol for which it may be considered as an effective alternative to the existing methodologies. The pronounced advantage of this novel catalytic system is expected to contribute to the development of more benign Pechmann condensation reactions of phenols with $\beta$-keto esters.

\section{ACKNOWLEDGEMENTS}

BT thanks the Department of Science and Technology, New Delhi, for a Research Fellowship under SERC Scheme (SP/S1/PC-31/2004). MKP is the recipient of Senior Research Fellowship of CSIR, New Delhi.

\section{REFERENCES}

[1] Weber, U.S.; Steffen, B.; Siegers, C.P. Res. Commun. Mol. Pathol. Pharmacol., 1998, 99, 193.

[2] Patil, A.D.; Freyer, A.J.; Drake, S.E.; Haltiwanger, R.C.; Bean, M.F.; Taylor, P.B.; Caranfa, M.J.; Breen, A.L.; Bartus, H.R.; Johnson, R.K.; Hertzberg, R.P.; Westley, J.W. J. Med. Chem., 1993, 36, 4131.

[3] Yun, B.S.; Lee, I.K.; Ryoo, I.J.; Yoo, I.D. J. Nat. Prod., 2001, 64 1238.

[4] Cheng, J.F.; Ishikawa, A.; Ono, Y.; Arrhenius, T.; Nadzan, A. Bioorg. Med. Chem., 2003, 13, 3647.

[5] Zaha, A.A.; Hazem, A. Microbiologica., 2002, 25, 213.

[6] Whittaker, M.; Floyd, C.D.; Brown, P.; Gearing, A.J.H. Chem. Rev., 1999, 99, 2735.

[7] Maly, D.J.; Leonetti, F.; Backes, B.J.; Dauber, D.S.; Harris, J.L.; Craik, C.S.; Ellman, J.A. J. Org. Chem., 2002, 67, 910.

[8] Pechmann, V.H.; Duisberg, C. Chem. Ber., 1884, 17, 929. 
[9] Johnson, J.R. Org. React., 1942, 1, 210.

[10] Brufola, G.; Fringuelli, F.; Piermatti, O.; Pizzo, F. Heterocycles, 1996, 43, 1257.

[11] Shirner, R.L. Org. React., 1942, $1,1$.

[12] Yavari, I.; Hekmat, S.R.; Zonouzi, A. Tetrahedron Lett., 1998, 39, 2391.

[13] Cairns, N.; Harwood, L.M.; Astles, D.P. J. Chem. Soc. Perkin Trans., 1994, 1, 3101 .

[14] Cartwright, G.A.; McNab, W. J. Chem. Res. (S), 1997, 296.

[15] Woods, L.L.; Sapp, J. J. Org. Chem., 1962, 27, 3703.

[16] Robertson, A.; Sandrock, W.F.; Henry, C.B. J. Chem. Soc., 1931, 2426.

[17] Sharma, G.V.M.; Janardhan, R.J.; Sree, L.P.; Radha, K.P. Tetrahedron Lett., 2005, 46, 6119.

[18] Valizadeha, H.; Shockravi, A. Tetrahedron Lett., 2005, 46, 3501.

[19] Potdar, M.K.; Mohile, S.S.; Salunkhe, M.M. Tetrahedron Lett., 2001, 42, 9285 .

[20] Rodriguez-Dominguez, J.C.; Kirsch, G. Tetrahedron Lett., 2006, 47, 3279 .

[21] Selvakumar, S.; Chidambaram, M.; Singh, A.P. Catal. Commun., 2007, 8, 777.

[22] Frere, S.; Thiery, V.; Besson, T. Tetrahedron Lett., 2001, 42, 2791.

[23] Chaudhari, D.A. Chem. Ind., 1983, 568.
[24] Naik, M.A.; Mishra, B.G.; Dubey, A. Colloids Surf. A Physicochem. Eng. Aspects, 2008, 317, 234

[25] Gunnewegh, E.A.; Hoefnagel, A.J.; van Bekkum, H. J. Mol. Catal. A: Chem., 1995, 100, 87.

[26] Fre're, S.; Thie'ry, V.; Besson, T. Tetrahedron Lett., 2001, 42, 2791

[27] Heravi, M.M.; Ajami, D.; Ghassemzadeh, M. Synth. Commun., 1999, 29, 1013.

[28] Oskooie, H.A.; Heravi, M.M.; Sadnia, A.; Jannati, F.; Behbahani, F.K. Monatsh. Chem., 2008, 139, 27.

[29] Wu, H.; Shen, Y.; Fan, L.-y.; Wan, Y.; Zhang, P.; Chen, C.-f.; Wang, W.-x. Tetrahedron, 2007, 63, 2404.

[30] Shaterian, H.R.; Ghashang, M.; Feyzi, M. Appl. Catal. A Gen., 2008, 345, 128.

[31] Mohammadpoor-Baltork, I.; Mirkhani, V.; Moghadam, M.; Tangestaninejad, S.; Zolfigol, M.A.; Abdollahi-Alibeik, M.; Khosropour, A.R.; Kargar, H.; Hojati, S.F. Catal. Commun., 2008, 9, 894.

[32] Reddy, B.M.; Patil, M.K.; Reddy, B.T. Catal. Lett., 2008, 125, 97.

[33] Reddy, B.M.; Patil, M.K.; Reddy, B.T.; Park, S.-E. Catal. Commun., 2008, 9, 950 .

[34] Reddy, B.M.; Patil, M.K.; Rao, K.N.; Reddy, G.K. J. Mol. Catal. A Chem., 2006, 258, 302.

[35] Reddy, B.M.; Patil, M.K.; Reddy, B.T. Catal. Lett., 2008, 126, 413.

(C) Reddy et al.; Licensee Bentham Open.

This is an open access article licensed under the terms of the Creative Commons Attribution Non-Commercial License (http:/creativecommons.org/licenses/by$\mathrm{nc} / 3.0 /$ ) which permits unrestricted, non-commercial use, distribution and reproduction in any medium, provided the work is properly cited. 